The great discrepancy between the old traditions and the modern Western way of life as disseminated by television, magazines, tourism and rapidly increasing education (with mixed sexes) is increasing tension among the young. They do not know how to behave. Even religious faith is weakening.

We observed that those who are educated are more prone to the 'cry for help' (68 per cent). They appear to be vulnerable to the conflict between old and new cultures, whereas simple people, especially those outside big cities, are more satisfied and content. The strong faith and cohesion of the traditional society are stabilizing factors. Another stress is the new law of compulsory education for those between 6 and 45 years of age, which has brought to notice those who cannot cope with the demands of schooling. The sick role can exempt from education on medical grounds.

TARIK I. HAMDI

College of Medicine, Al-Mustansiriyah University, Baghdad, Iraq

Al-Yarmouk Teaching Hospital,

L. Al-Hasani

A. MAHMOOD Baghdad

Neuropsychiatric Unit, College of Medicine, Baghdad University

\section{A. Al-Husaini}

\section{OILY INJECTIONS THAT OOZE}

\section{DEAR SiR,}

Some observations of an experienced nurse on the technique and results of giving depot injections for schizophrenia might help patients, nurses and doctors. I have noticed, and you can easily confirm this, that some of the oily injection frequently leaks out of the puncture in the skin, soiling clothes or sticking plaster. Put plasters on injection punctures and you will see what I mean. Sometimes these leaks must be a significant proportion of the volume of drug originally injected, and I strongly suspect that this is the explanation of the comment frequently heard in psychiatric wards, "the injection never touched her".

Years ago, leaking and staining was a common problem with intramuscular injections of iron. Many nurses nowadays have forgotten, or never been taught, the technique used then of sliding the skin to one side before the injection and then back afterwards, sealing the track of the needle. It works.

Catherine Finlay KinNes

96 Huntley Drive,

Tanshall, Glenrothes,

Fife $\mathrm{K} Y \mathrm{O} 2 \mathrm{HT}$

\section{NORADRENERGIC OVER-ACTIVITY IN CHRONIC SCHIZOPHRENIA}

DEAR SIR,

U. C. R. Gomes and colleagues (Journal, October $1980,137,346-51)$ claim that statistical analyses eliminated the possibility that their finding of increased noradrenaline and cycle AMP in the cerebrospinal fluid of chronic schizophrenics was related to neuroleptic medication. They explained that the Wilcoxon test was applied to patients receiving medication who were matched for (inter alia) diagnosis, with patients not receiving medication.

How was this possible, given that Table I showed that all their chronic schizophrenic patients were receiving medication?

\section{E. R. Squibb and Sons Limited, Squibb House, 141-149 Staines Road, Hounslow TW3 3JB}

Dear Sir,

Dr Schiff has quite correctly pointed out that all the chronic schizophrenic patients in our study were on neuroleptic medication. It was therefore not possible to make direct comparisons of medicated and unmedicated patients in this category, nor did we claim to have done so.

Consequently, we were compelled to examine the effects of medication on CSF noradrenaline and cyclic AMP concentration in the other three groups of psychiatric patients, i.e. those with acute schizophrenia, psycho-organic disorders and personality disorders. Our findings clearly showed that neuroleptic medication made no significant difference to any of the parameters studied. We therefore feel fully justified in our interpretation that ". . . neither the elevated noradrenaline nor cyclic AMP concentrations in the chronic schizophrenics were attributable to drug effects".

Department of Biochemistry,

B. C. Shanley

\section{University of Queensland,}

St Lucia, Australia 4067

\section{IDENTIFICATION OF DISEASE ENTITIES}

DEAR SIR,

Even Kendell and Brockington's paper (Journal, 137, 324-31), which doesn't go far enough, makes us pose the question of when is it appropriate to discuss whether psychiatric terms refer to dimensions or categories. The idea that a mathematical technique could resolve it is a philosophical mistake, one which will obscure rather than clarify real issues. Any two 